\title{
Integrated Magnetometry Platform with Stackable Waveguide-Assisted Detection Channels for Sensing Arrays
}

\author{
Michael Hoese $\odot,{ }^{1, \dagger}$ Michael K. Koch $\odot,{ }^{1,2, \dagger}$ Vibhav Bharadwaj $\odot,{ }^{3}$ Johannes Lang, ${ }^{1}$ \\ John P. Hadden $\odot,{ }^{4}$ Reina Yoshizaki®, ${ }^{5}$ Argyro N. Giakoumaki, ${ }^{3}$ Roberta Ramponi, ${ }^{3}$ Fedor Jelezko, ${ }^{1,2}$ \\ Shane M. Eaton, ${ }^{3}$ and Alexander Kubanek $\oplus^{1,2, *}$ \\ ${ }^{1}$ Institute for Quantum Optics, Ulm University, Ulm D-89081, Germany \\ ${ }^{2}$ Center for Integrated Quantum Science and Technology (IQst), Ulm University, Ulm D-89081, Germany \\ ${ }^{3}$ Institute for Photonics and Nanotechnologies (IFN) - CNR, Piazza Leonardo da Vinci, 32, Milano 20133, Italy \\ ${ }^{4}$ School of Physics and Astronomy, Cardiff University, Cardiff CF24 3AA, United Kingdom \\ ${ }^{5}$ Department of Mechanical Engineering, School of Engineering, The University of Tokyo, Tokyo 113-8656, Japan
}

(Received 22 December 2020; revised 26 April 2021; accepted 10 May 2021; published 26 May 2021)

\begin{abstract}
The negatively charged nitrogen vacancy $\left(\mathrm{N}-V^{-}\right)$center in diamond has shown great success in nanoscale, high-sensitivity magnetometry. Efficient fluorescence detection is crucial for improving the sensitivity. Furthermore, integrated devices enable practicable sensors. Here, we present an integrated architecture which allows us to create $\mathrm{N}-V^{-}$centers a few nanometers below the diamond surface, and at the same time covering the entire mode field of femtosecond-laser-written type-II waveguides. We experimentally verify the coupling efficiency, showcase the detection of magnetic resonance signals through the waveguides and perform proof-of-principle experiments in magnetic field and temperature sensing. The sensing task can be operated via the waveguide without direct light illumination through the sample, which is important for magnetometry in biological systems that are sensitive to light. In the future, our approach will enable the development of two-dimensional sensing arrays facilitating spatially and temporally correlated magnetometry.
\end{abstract}

DOI: 10.1103/PhysRevApplied.15.054059

\section{INTRODUCTION}

Quantum sensing performed with negatively charged nitrogen vacancy $\left(\mathrm{N}-V^{-}\right)$centers in diamond has successfully measured strain [1], temperature [2], and magnetic fields [3-5] at the nanoscale and with high sensitivity. $\mathrm{N}-V^{-}$-based magnetometry is widely applied to characterize biological samples such as cells [6] or complex materials $[7,8]$. The combination with atomic-force microscopy has revolutionized scanning-probe magnetometry $[9,10]$ with applications in material science and life sciences. Advanced photonics could further improve the performance in terms of losses, signal-to-noise, and operation speed [11,12] and includes integration into optical fibers $[13,14]$, diamond nanopillar arrays $[15,16]$, and integrated photonics [17-19]. The latter enables, in addition, the realization of compact devices. In this context, femtosecond laser writing is an outstanding fabrication method that does not rely on lithography steps and has, in particular, the capability to fabricate three-dimensional structures. Using diamond as the photonics host offers the

\footnotetext{
${ }^{*}$ Corresponding author. alexander.kubanek@uni-ulm.de

${ }^{\dagger}$ These authors contributed equally to this work.
}

additional advantage that $\mathrm{N}-\mathrm{V}^{-}$centers can be integrated directly into the photonic device. Laser-written type-II waveguides in diamond have recently been developed $[20,21]$ and show great potential to efficiently interface $\mathrm{N}-V^{-}$centers. Thereby, a focused laser beam creates two nearby lines of reduced refractive index, where the stressed region between the two lines serves as a waveguide. The controlled creation of defect centers has been demonstrated with ion implantation [22] and laser writing [2325]. However, until now there has been no strategy that enables deterministic postprocessing of each waveguide in a three-dimensional platform, a problem that becomes even more challenging when color centers are required close to the diamond surface as is the case for $\mathrm{N}-V^{-}$-based magnetometry.

Here, we present an integrated approach that is capable of functionalizing each waveguide in a three-dimensional architecture individually with $\mathrm{N}-\mathrm{V}^{-}$centers as depicted in Fig. 1(a). After laser writing the waveguides, we create $\mathrm{N}-V^{-}$centers through shallow implantation of nitrogen ions on the front facet of the diamond photonics platform. The implantation depth of a few nanometers below the diamond surface enables the combination of efficient photon routing through diamond waveguides with sensing tasks on 

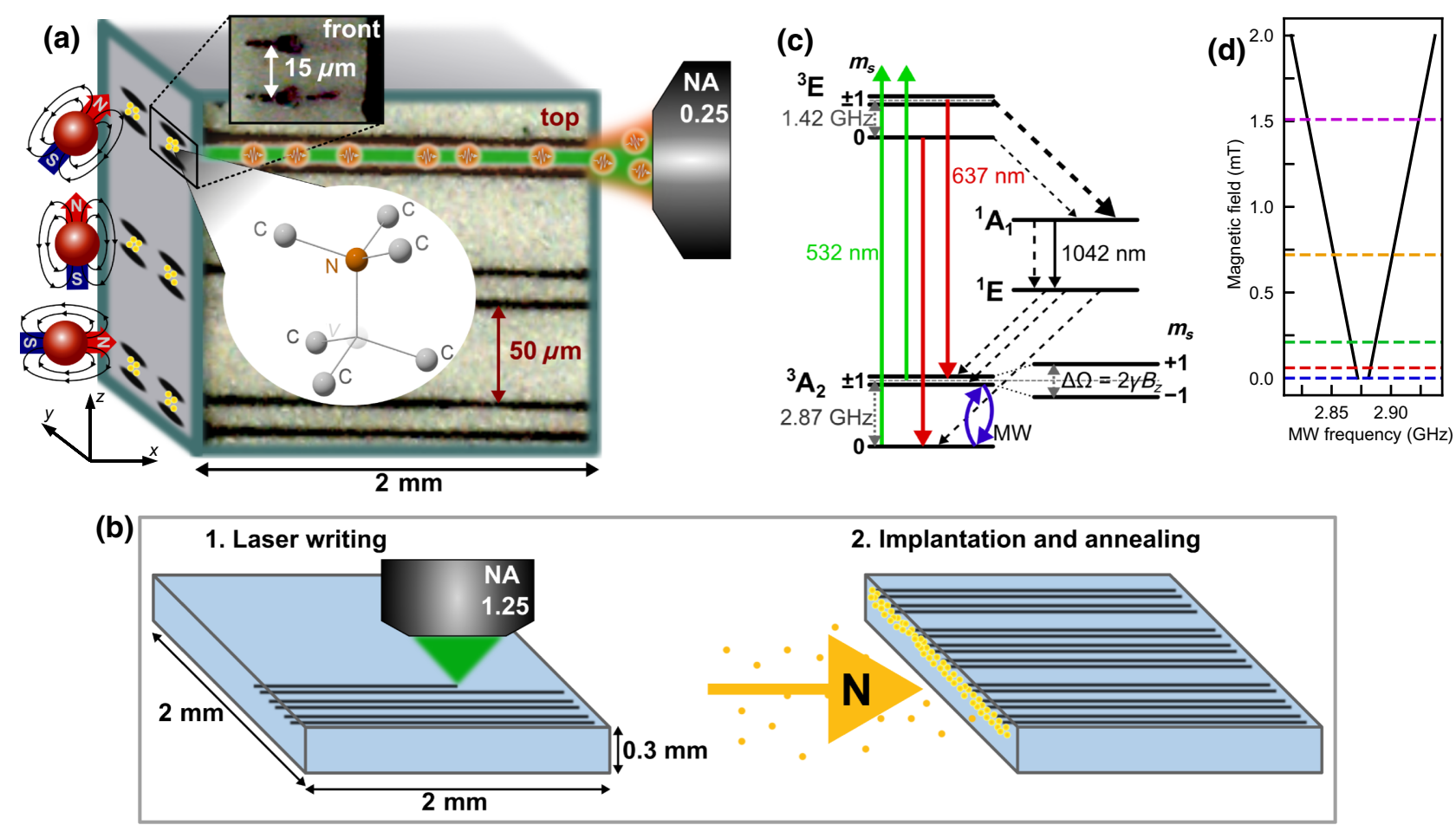

FIG. 1. (a) Sketch of the waveguide-assisted sensor array. Shallow-implanted $\mathrm{N}-V^{-}$centers act as local magnetometers on the diamond surface. The $\mathrm{N}-V^{-}$centers are optically accessed by means of laser-written type-II waveguides and low-numerical-aperture (low-NA) optics. The architecture can be extended to sensor arrays. The sketch incorporates microscope images of the waveguides in top and front view. (b) Sample fabrication process. First, the waveguide walls (black lines) are laser written from the top into the diamond. Second, nitrogen ions are shallow implanted into the front facet to form $\mathrm{N}-V^{-}$centers after annealing. (c) Level scheme and dynamics of the $\mathrm{N}-V^{-}$center as utilized to perform optically detected magnetic resonance (ODMR) spectroscopy. Dashed arrows depict phonon transitions and bold arrows photon transitions, respectively. The arrow width reflects the interaction strength. (d) Model for the splitting of the $m_{s}= \pm 1$ ground states with increasing magnetic field. The dashed lines indicate the magnetic field strengths that we apply in the experiments to perform ODMR scans.

the diamond surface. Our architecture separates the optical access to the $\mathrm{N}-V^{-}$centers from the object to be sensed by excitation and detection through the waveguides, in a similar way to demonstrations based on total internal reflection $[26,27]$. Therefore, as with common confocal detection [28] and widefield imaging [29] approaches, good sensing performance can be achieved without the need of light exposure through the sample. However, the thickness of the diamond sample can be much larger in our case. Ultimately, the architecture can be extended to multiple, individually addressable waveguides, thus forming a twodimensional sensing array with potential for time-resolved and spatially correlated magnetometry in material and life sciences.

In the following, we discuss the working principle of our sensing device and characterize the waveguide-assisted optical access. Then, we perform proof-of-principle magnetic field and temperature sensing with ensembles of $\mathrm{N}-V^{-}$centers. We compare the sensor performance with respect to the state of the art.

\section{WORKING PRINCIPLE}

Type-II waveguides are written in a chemical vapor deposition (CVD) grown electronic-grade diamond slab $(2 \mathrm{~mm} \times 2 \mathrm{~mm} \times 0.3 \mathrm{~mm})$ with pulsed laser illumination at $515 \mathrm{~nm}$, with a repetition rate of $500 \mathrm{kHz}$, pulse width of $300 \mathrm{fs}$ and laser power of $100 \mathrm{~mW}$. The laser beam is focused into the sample through a high-numerical-aperture (high-NA) objective $(1.25 \mathrm{NA}, 100 \times)$ to create type-II waveguides of $2 \mathrm{~mm}$ length, according to the size of the diamond, in depths ranging from 5 to $25 \mu \mathrm{m}$ below the top diamond surface. The femtosecond laser writing damages the diamond lattice structure within the modification lines serving as waveguide walls. This causes a reduced refractive index at the waveguide walls and an increased refractive index between two walls due to stress in the diamond lattice. Hence, the waveguide mode is confined to the center between two waveguide walls for light guiding [30]. The waveguide depths are measured from the surface to the center of the modification. The type-II waveguide width of $15 \mu \mathrm{m}$ [center-to-center 
transverse spacing between the two laser modification tracks as shown in Fig. 1(a) front view] is optimized for single-mode, low-loss light transmission between $630 \mathrm{~nm}$ and $740 \mathrm{~nm}$ according to the $\mathrm{N}-V^{-}$-center sideband emission. In order to functionalize the waveguides with $\mathrm{N}-V^{-}$ centers, we shallow implant Nitrogen ions into the front facet of the waveguides followed by subsequent annealing at $1000^{\circ} \mathrm{C}$. Further details on the fabrication can be found in Fig. 1(b) and in the methods section.

The spin state of the $\mathrm{N}-V^{-}$center, a spin-1 system in its ground and excited states, can be detected optically via magnetic resonance spectroscopy (ODMR) [31], as sketched in Fig. 1(c). Shelving from $m_{s}= \pm 1$ states induces optical pumping and allows to read out the state via measurement of the fluorescence intensity. The microwave (MW) resonance frequency decreases from $2.88 \mathrm{GHz}$ at cryogenic temperatures to $2.87 \mathrm{GHz}$ at room temperature thereby enabling, in principle, temperature sensing over a wide temperature range although the sensitivity decreases at low temperatures. In a magnetic field, the degeneracy of the $m_{s}= \pm 1$ states lifts due to a magnetic-field-dependent splitting of $\Delta \Omega=2 \gamma B_{z}$, with the gyromagnetic ratio $\gamma=$ $28 \mathrm{GHz} \mathrm{T}{ }^{-1}$, of the two states. As a result, the ODMR resonance dip splits into two, one for each spin state, thus enabling inference of the magnetic field strength. Figure 1(d) illustrates the magnetic field dependent splitting. Here, the degeneracy is already lifted at zero-field owing to residual strain in the diamond lattice [32,33]. For continuous-wave ODMR (CW ODMR) measurements, the overall sensitivity to dc magnetic fields [34],

$$
\eta_{\mathrm{dc}}=\frac{\hbar}{g_{e} \mu_{B} \sqrt{N \tau}}
$$

depends on the number of $\mathrm{N}-V^{-}$centers $N$ with coherence time $\tau$. Thereby, $g_{e}=2.0$ denotes the electron $g$ factor, $\mu_{B}$ the Bohr magneton and $\hbar$ the Planck constant. One way to improve the overall sensitivity is to increase $N$. This can either be done by increasing the density of $\mathrm{N}-V^{-}$centers or by enlarging the sensing volume. However, progressing to large sensing volumes remains challenging. The efficient extraction of photons out of the diamond host from a large ensemble of $\mathrm{N}-V^{-}$centers distributed over a large sensing area is an outstanding problem. We therefore begin with the benchmarking of the device detection efficiency with respect to conventional, well-established confocal ODMR measurements. We then extrapolate the overall sensitivity of the device by taking into account the large $\mathrm{NV}^{-}$ ensemble that is addressable via the waveguide mode.

\section{WAVEGUIDE-ASSISTED OPTICAL ACCESS}

We first compare the detected signal through the waveguide with the confocal detection. Therefore, we excite a confocal spot of the $\mathrm{N}-V^{-}$ensemble and simultaneously measure the transmission signal through the waveguide and the confocal signal in reflection. We examine the transmission properties for waveguides at a depth of $20 \mu \mathrm{m}$ below the diamond surface with well-isolated waveguide modes. A confocal scan of the waveguide front facet, depicted in Fig. 2(a), shows the bright areas where $\mathrm{N}-V^{-}$centers are created. The areas around waveguide walls appear brighter indicating an increased number of $\mathrm{N}-V^{-}$centers. In this study, we focus on the leftmost waveguide (green box). To characterize the transmission of the waveguide, we map the mode excitation shown in Fig. 2(b) by laterally scanning a $738 \mathrm{~nm}$ laser while recording the maximum transmission signal. The 2D-Gaussian mode profile is projected onto the horizontal and vertical coordinate axes. We obtain a similar mode profile when exciting the $\mathrm{N}-V^{-}$centers with $532 \mathrm{~nm}$ laser light and collecting the fluorescence in transmission through the waveguide while laterally scanning the excitation laser [see Fig. 2(c)]. We note that the transmitted fluorescence resembles the mode profile, although the front facet is homogeneously covered with $\mathrm{N}-V^{-}$centers [see the confocal scan in Fig. 2(a)]. We conclude that $\mathrm{N}-\mathrm{V}^{-}$emission is guided through the waveguide.

In order to benchmark the detection efficiency of the waveguide-assisted optical access, we first compare the photoluminescence (PL) spectrum from the $\mathrm{N}-V^{-}$ensemble in confocal configuration (reflection) with the spectrum acquired in transmission through the waveguide (see Fig. 2(d) for experiment schematic) as shown in Fig. 2(e). Note that in order to clearly determine the $\mathrm{N}-V^{-}$emission we cooled the sample to cryogenic temperatures. The resulting PL spectra, both in transmission and reflection, reveal the characteristic zero-phonon line (ZPL) and phonon-sideband (PSB) emission of $\mathrm{N}-V^{-}$centers. Here, intensities are normalized with respect to the ZPL emission for sake of clarity. The first thing to note is that the polarization pattern of the waveguide mode, shown in the inset of Fig. 2(b), yields a distinct polarization contrast which matches the polarization of the waveguided $\mathrm{N}-V^{-}$fluorescence within $\approx 16.5^{\circ}$. From the measured transmission and reflection spectra we extract the ODMR-signal detection efficiency of our waveguide-assisted sensor with respect to conventional confocal measurements.

Figure 2(f) illustrates the ratio of transmitted signal versus confocal detection which we refer to as relative detection efficiency. The relative efficiency evidences the challenge to extract photons from a large detection volume with respect to a resolution-limited spot. The waveguide transmission losses are disregarded in this comparison and will be considered later together with the absolute detection efficiency. The green curve marks the detection efficiency for a $\mathrm{N}-V^{-}$subensemble that is not perfectly centered compared with the waveguide mode (see Fig. 2(c) green circle and dashed white cross for the excitation point), resulting in a reduction of the intensity by the factor 


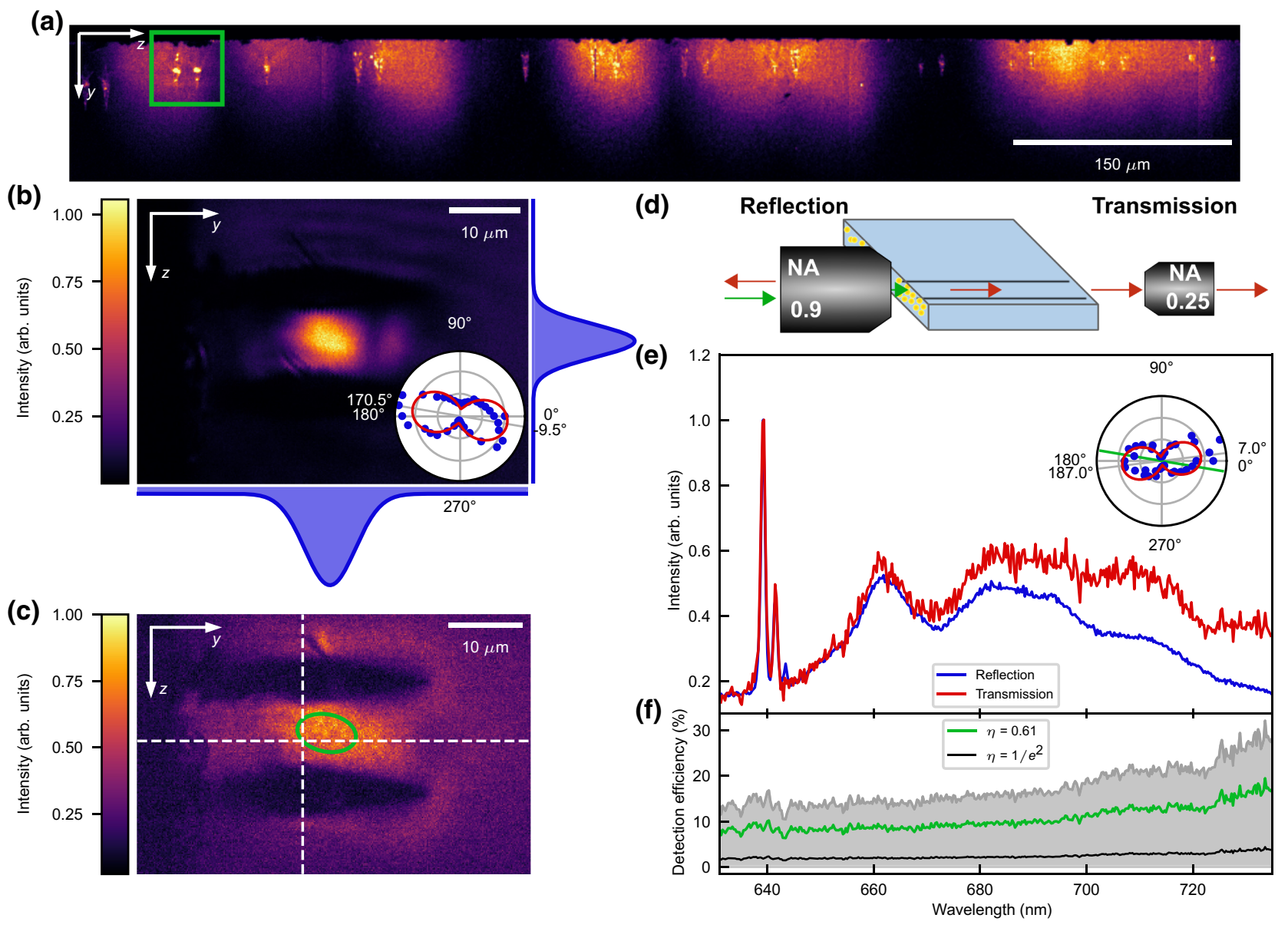

FIG. 2. (a) Confocal scan showing the fluorescence spots of the shallow-implanted N- $V^{-}$centers on the front facet of the sample. The leftmost waveguide (marked with a green box) is studied in the following. (b) Mode shape of the waveguide (WG). The shape of the WG mode is revealed by laterally scanning a $738 \mathrm{~nm}$ laser while recording the collected counts in transmission. The two intersections at the side show the corresponding 2D Gaussian fit to the WG mode. The corresponding mode-polarization contrast is shown in the lower right corner. Note that the image is rotated by $90^{\circ}$ with respect to panel (a). (c) $\mathrm{N}-V^{-}$transmission through the waveguide. We excite the N- $V^{-}$centers off-resonantly with $532 \mathrm{~nm}$ laser light from the front and collect the transmitted signal. Laterally scanning the excitation laser resembles the waveguide mode in transmission. The intersection of the dashed lines denotes the position which is at $61 \%$ of the maximum of the WG mode (green ellipse). (d) Experiment schematic. The sketch illustrates the two detection channels, reflection and transmission. (e) The PL spectra in reflection and transmission are compared. The exposure time of the transmission measurement is ten times larger than for the measurement in reflection. Both spectra are normalized to the ZPL. The inset shows the ZPL polarization measured in transmission through the waveguide, which resembles the waveguide mode polarization (marked as a green line). (f) We extract the wavelength-specific optical detection efficiency of the N- $V^{-}$ensemble to the waveguide mode by comparing the transmitted PL spectrum with the fluorescence in reflection and by accounting for losses that occur until detection. The green curve is the inferred efficiency for a $\mathrm{N}-V^{-}$ensemble located at $61 \%$ of the optimal coupling corresponding to the green ellipse in (c). The black lines marks the $1 / e^{2}$-profile of the Gaussian mode.

0.61 compared with the maximum at the optimal position. We calculate relative detection efficiencies ranging from $8 \%$ at $660 \mathrm{~nm}$ to more than $15 \%$ at $735 \mathrm{~nm}$. The gray curve gives the efficiency for an ideally coupled $\mathrm{N}-\mathrm{V}^{-}$center located at the maximum of the waveguide mode, yielding ideal relative detection efficiencies up to $30 \%$ at $735 \mathrm{~nm}$. The gray shaded area indicates all possible detection efficiencies from ideally to completely uncoupled ensembles. The black line marks the $1 / e^{2}$ profile of the Gaussian mode that will be excited when operating the sensor through the waveguide. The figure illustrates the large sensing area of up to $105 \mu \mathrm{m}^{2}$ per waveguide mode with an average relative detection efficiency of $10.4 \%$ for the large ensemble of $\mathrm{N}-V^{-}$centers which is addressed via the waveguide mode.

We now quote the absolute detection efficiency taking into account the waveguide transmission losses including the outcoupling efficiency of up to $79.8 \%$, or $6.95 \mathrm{~dB}$, which is comparable to values reported for type-II waveguide in diamond $[20,35]$. The absolute detection efficiency of the confocal collection behind the objective is $2.5 \%$ 
corresponding to an absolute detection efficiency of $0.05 \%$ through the waveguides when averaged over the $1 / e^{2}$ area of the Gaussian mode. The waveguide detection efficiency corresponds to a waveguide coupling efficiency of $0.3 \%$ in agreement with estimated coupling efficiencies for individual $\mathrm{NV}^{-}$centers in laser-written waveguides [24] of $0.1 \%$. In future designs, shorter waveguides and improved outcoupling performance will increase transmission, thus leading to higher detection efficiencies.

Although the detection efficiency of a confocally excited $\mathrm{N}-V^{-}$-center ensemble, as expected, decreases with respect to conventional confocal detection, the large number of addressable $\mathrm{N}-V^{-}$centers via the waveguide mode increases the achievable overall sensitivity. We extrapolate the increased number of $\mathrm{N}-\mathrm{V}^{-}$centers that is accessible via the waveguide mode by comparing the mode field area of $105 \mu \mathrm{m}^{2}$ with the confocal spot size of $0.062 \mu \mathrm{m}^{2}$. Taking into account the homogeneous distribution of $\mathrm{N}-V^{-}$centers over the entire waveguide mode, we expect an increase in the number of addressed $\mathrm{N}-V^{-}$centers $N$ by a factor of 1690. Compared with conventional confocal detection, the sensitivity thus improves by a factor of 41 according to the $\sqrt{N}$ dependency.

\section{SENSOR PERFORMANCE}

In the following, we study the sensing performance of our device. All measurements are performed at room temperature although the sensor can be operated over a wide temperature range. We excite the $\mathrm{N}-V^{-}$ensemble with offresonant $(532 \mathrm{~nm})$ laser light, which is coupled from the back side into the waveguide. The $\mathrm{N}-V^{-}$signal is also read out through the waveguide in order to keep the front facet of the diamond sensing area, which contains the shallowimplanted $\mathrm{N}-V^{-}$ensemble, fully accessible. Figure 3(a) shows a confocal scan of the diamond backside with the low-NA objective used for incoupling of excitation light and read-out of the $\mathrm{N}-V^{-}$signal through the waveguide. The waveguide mode is clearly resolved including the waveguide walls confining the mode, which appear dark. The spectrum of the waveguide-assisted emission resembles the spectrum of the $\mathrm{N}-V^{-}$ensemble, as highlighted by comparison with a standard confocal spectrum of the ensemble in Fig. 3(b). The background spectrum is shown in red and is recorded spatially shifted from the waveguide mode.

We now utilize the $\mathrm{N}-\mathrm{V}^{-}$ensemble as a magnetic field sensor by exploiting its ODMR signal. Therefore, we apply a MW field to the sample and vary the external magnetic field by approaching the sensor with a permanent magnet. Varying the MW frequency reveals the characteristic dips in the fluorescence signal, as shown in Fig. 3(c). First, we compare the ODMR signal at zero magnetic field detected in standard confocal configuration with the transmission through the waveguide. Both signals reveal two characteristic dips separated by $9 \mathrm{MHz}$ due to strain induced by the laser-written waveguides [30], residual strain in the diamond lattice $[32,33]$ and also background magnetic field from the environment. An applied magnetic field increases the splitting between the two dips as shown in Fig. 3(c), both in transmission and reflection [see Fig. 2(d) for a schematic of the experiment]. Figure 3(d) shows the increased splitting with increasing magnetic field strength. At higher magnetic fields, each dip splits into two, because the $\mathrm{N}-\mathrm{V}^{-}$centers experience different magnetic fields depending on their orientation in the crystal lattice [36]. We use the gyromagnetic ratio of an $\mathrm{N}-\mathrm{V}^{-}$ center, $\gamma=\left(g_{e} \mu_{B} / \hbar\right)=28 \mathrm{GHz} \mathrm{T}{ }^{-1}$, in order to calculate the splitting due to an applied magnetic field $B$ along the $\mathrm{N}-V^{-}$center axis as $\Delta \Omega=2 \gamma B_{z}$ (see [37]). From the spin Hamiltonian of the $\mathrm{N}-V^{-}$center,

$H=D\left(S_{z}^{2}-\frac{1}{3}[S(S+1)]\right)+E\left(S_{x}^{2}-S_{y}^{2}\right)+g_{\mathrm{e}} \mu_{\mathrm{B}} \mathbf{B} \cdot \mathbf{S}$,

we deduce the magnetic field [5] as

$$
\left(g_{\mathrm{e}} \mu_{\mathrm{B}} B\right)^{2}=\frac{1}{3}\left(v_{1}^{2}+v_{2}^{2}-v_{1} v_{2}-D^{2}\right)-E^{2} .
$$

The zero-field splitting (ZFS) parameter is given as $D$ and a nonzero $E$ incorporates additional splitting of the two dips, located at MW frequencies $v_{1}$ and $v_{2}$ at zero magnetic field due to strain. The ZFS could be further increased by the Earth's magnetic field. In our case, the ODMR measurements yield effective magnetic fields between 0.066 and $1.508 \mathrm{mT}$ along the $\mathrm{N}-V^{-}$center axis, respectively.

Our measurements showcase the ability to measure magnetic fields with a precision better than $6 \mu \mathrm{T}$ with our device. The lowest panel of Fig. 3(d) illustrates the fitted peak positions together with their error margins that are now used to estimate the precision of magnetic field measurements. The two measurements are performed at 0.066 and $0.214 \mathrm{mT}$ applied magnetic field, respectively, with two clearly separated peak positions. Their fit error margins are still significantly separated. We calculate the error of the magnetic field difference of the two measurements including their fit errors. With these fit errors as reference, we estimate that magnetic field differences of $6 \mu \mathrm{T}$ can be resolved with our device. The sensitivity $\eta_{\mathrm{dc}}$ of our magnetometer device to dc magnetic fields for CW ODMR measurements can be calculated as [38]

$$
\eta_{\mathrm{dc}}=\frac{\Delta v}{\gamma C \sqrt{I_{\mathrm{PL}}}}
$$

where $\Delta v$ denotes the full-width-at-half-maximum (FWHM) linewidth of the ODMR dip, $C$ denotes the ODMR contrast and $I_{\mathrm{PL}}$ the detected PL intensity in 


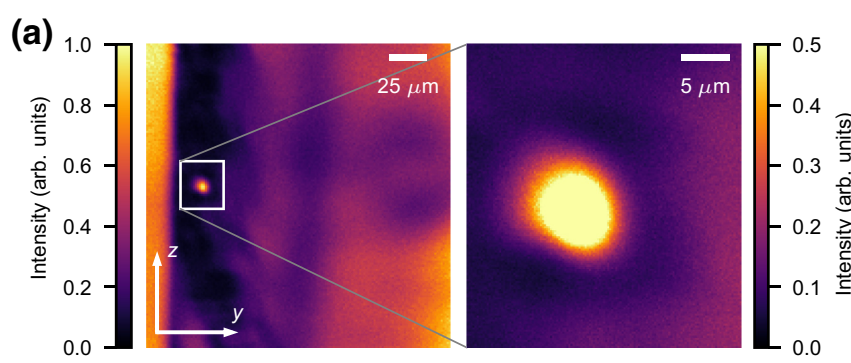

(b)

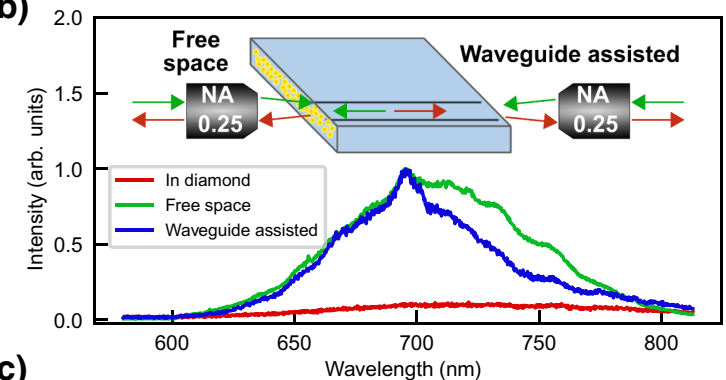

(c)

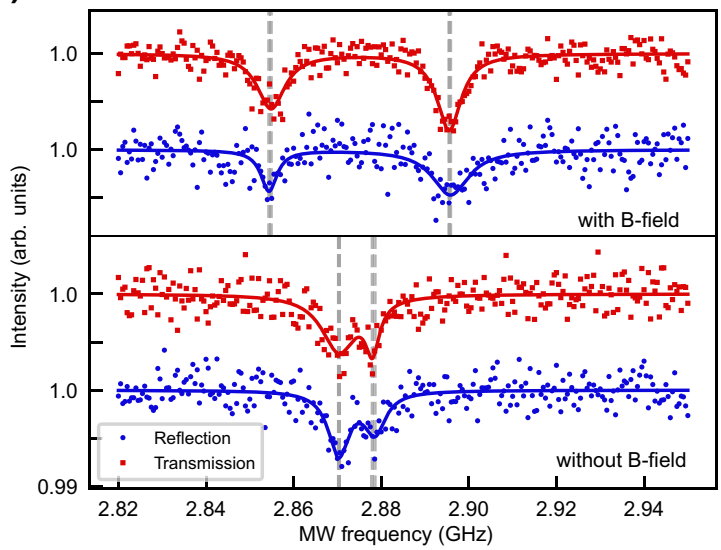

(d)

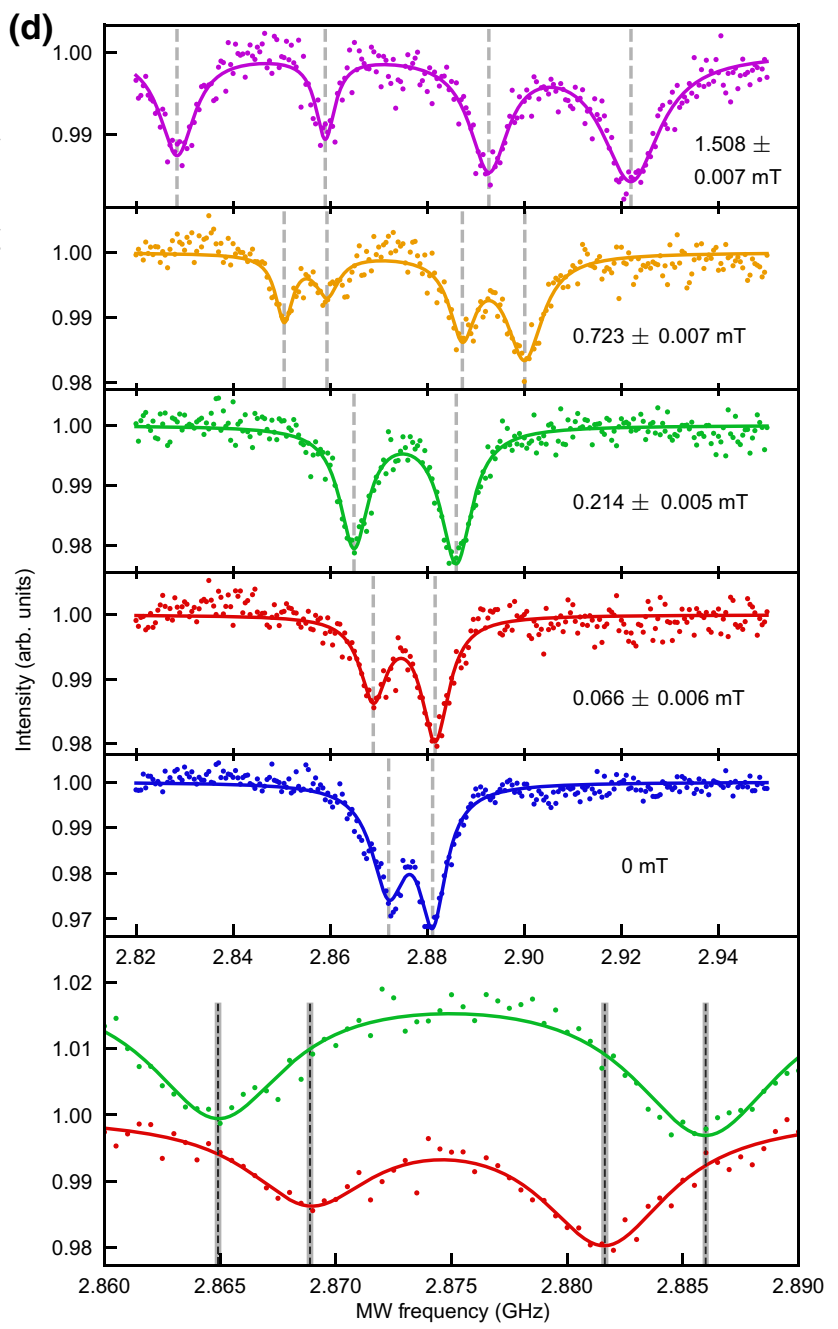

FIG. 3. (a) Confocal scan of the backside of the sample with a low-NA objective and an enlarged view of the waveguide mode. (b) Comparison of the PL spectra in transmission (blue) and reflection (green) of the waveguide mode as well as the transmitted signal outside the waveguide mode which we assign to the background signal (red). The inset shows the experimental setup used for the measurement. (c) Comparison of the ODMR spectrum at zero magnetic field and with applied magnetic field detected in confocal configuration (blue, reflection) and in transmission through the waveguide (red, transmission). (d) ODMR scans with varying magnetic field strengths between 0 and $1.508 \mathrm{mT}$. The lowest plot is an enlarged view comparing the ODMR scans at 0.066 and $0.214 \mathrm{mT}$. We infer the magnetic field sensitivity from the accuracy of the resonance frequency extracted from the Lorentzian fits.

counts per second. In our typical 30 min CW ODMR measurements, we reach sensitivities from Eq. (4) up to $36 \mu \mathrm{T} \mathrm{Hz}^{-1 / 2}$ in confocal configuration and $62 \mu \mathrm{T} \mathrm{Hz}$ when detecting the ODMR signal through waveguide transmission, which correspond to FWHMs of $7.5 \mathrm{MHz}$ in both cases. Hereby, different count rates and PL contrasts lead to differing sensitivities. These sensitivities are similar to hybrid approaches creating integrated quantum sensors $[13,14]$ or efforts towards miniaturization [39]. In addition, the above-stated precision better than $6 \mu \mathrm{T}$ corresponds to a sensitivity of $165 \mu \mathrm{T} \mathrm{Hz}{ }^{-1 / 2}$, which is larger because four frequency measurements contribute here. In the future, the sensitivity can be increased when using lock-in, pulsed ODMR, or Ramsey spectroscopy techniques [40].
Moreover, our measurement device works reliably over more than $20 \mathrm{~h}$ and the minimal measurement times for a full MW frequency scan can be reduced to less than $40 \mathrm{~s}$ with our settings [41].

We now investigate the performance of our device as a temperature sensor by exploiting the temperature dependence of the ZFS. Therefore, we heat the sample from room temperature $(294 \mathrm{~K})$ up to $324 \mathrm{~K}$ and measure ODMR without applying an external magnetic field. To analyze the change of the axial and transverse ZFS parameters, $D$ and $E$, we follow studies of $\mathrm{N}-V^{-}$ensembles in bulk diamond [42]. Linear fits to the data [see Fig. 4(a)], which serve as trend lines, reveal gradients of $(d D / d T)=$ $-40 \pm 18 \mathrm{kHz} \mathrm{K}^{-1}$ and $(d E / d T)=-8 \pm 15 \mathrm{kHz} \mathrm{K}{ }^{-1}$, 


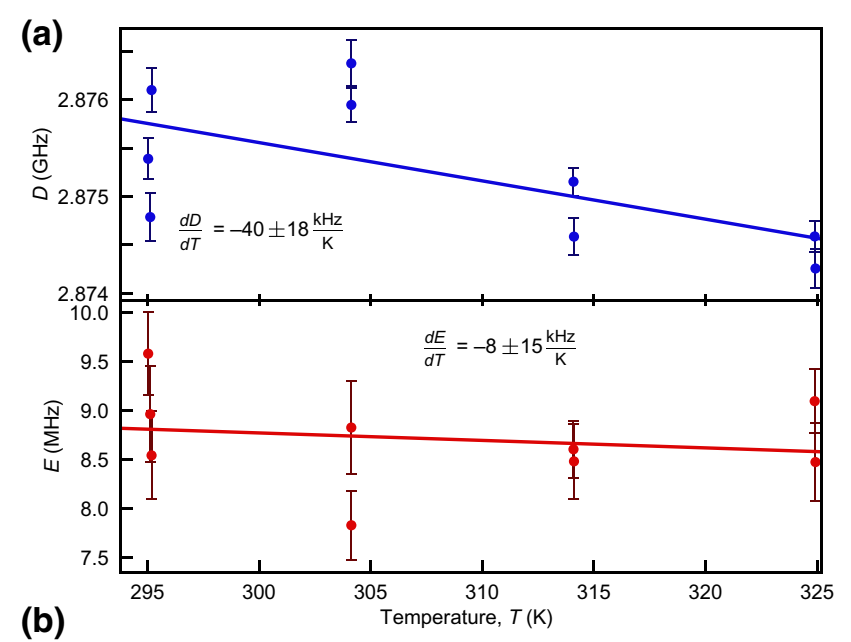

(b)

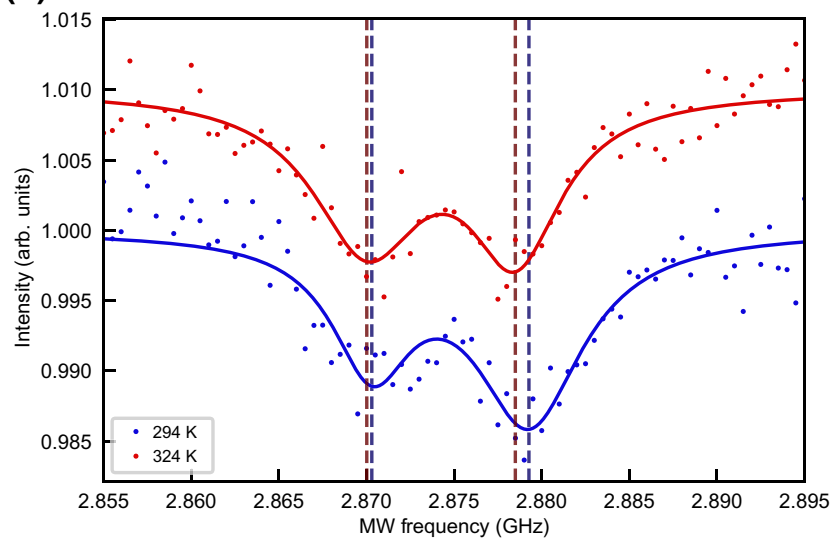

FIG. 4. (a) Temperature $T$ dependence of the axial and transverse ZFS parameters $D$ and $E$. (b) ODMR scans at temperatures of 294 and $324 \mathrm{~K}$ are used to illustrate temperature sensitivity of the ZFS parameters.

respectively. Here $d D / d T$ deviates from the values given in the literature [42] by roughly a factor of 1.5 . We tentatively ascribe this difference to a nonlinear systematic temperature difference between the diamond sensor and the heater (together with the heat sensor) below the sample holder. Thereby, we systematically overestimate temperatures larger than $294 \mathrm{~K}$. However, the large spread between measurements at the same temperature and resulting large fit error in the trend of $d D / d T$ makes a clear determination difficult. As strain typically only slightly affects $d D / d T$ (see [43]), we disregard strain as a possible explanation. Further, the trend of $d E / d T$ is dominated by a large fit error preventing any statement despite the similarity with studies in the literature [42]. A comparison of ODMR measurements at different temperatures underpins the temperature dependence [see Fig. 4(b)]. Note that the accessible temperature range for our device is only limited by the heating capabilities in our current setup and potentially covers the scale from high temperatures to cryogenic temperatures.

\section{DISCUSSION AND OUTLOOK}

Summarizing, we present an architecture for an integrated magnetometer based on $\mathrm{N}-V^{-}$centers implanted shallow in the field maximum of femtosecond-laserwritten type-II waveguides in diamond. We benchmark the waveguiding and coupling efficiency of the $\mathrm{N}-V^{-}$centers in transmission as well as the performance of the platform as magnetic field and temperature sensor. We benchmark the magnetic field sensitivity to at least $36 \mu \mathrm{T} \mathrm{Hz}^{-1 / 2}$. Nevertheless, nanotesla and picotesla sensitivities in bulk diamond with confocal [44-46], widefield [47,48], or integrated detection [26,49] provide a perspective for future improvements regarding $\mathrm{N}-V^{-}$-center creation and ODMR detection techniques, for example operating at the highest sensitivity point (at the maximum slope of the ODMR line). Furthermore, we expect an increased collection efficiency of $\mathrm{N}-V^{-}$-center emission, similar to photonic structures such as nanopillars [50], when waveguide losses are minimized in future experiments. The waveguide-assisted excitation and detection enables separation of the sensor operation from the sensing task making samples accessible that are sensitive to light illumination, such as paramagnetic labels and biomolecules that are not photostable. The device is compatible with a large temperature regime from cryogenic temperatures to high temperatures. The access to cryogenic temperatures could prove versatile when studying open questions in material science such as superconductivity.

Furthermore, due to the three-dimensional laser-writing capabilities [51-53], the sensor can be extended to twodimensional sensor arrays. The large area with accessible $\mathrm{N}-V^{-}$centers can be used in the future to achieve large filling factors of two-dimensional sensing arrays. The $1 / e^{2}$ mode area on the front facet covers about one half of the total waveguide area. Assuming densely packed lines of waveguides, where two horizontal waveguides share one laser-written side-wall and two vertical waveguides are slightly spaced to avoid coupling, results in overall filling factors on the sensing surface of about 0.2 . This proposed layout corresponds to pixel densities of $30 \mathrm{~mm}^{-1}$ in vertical $(y)$ and $67 \mathrm{~mm}^{-1}$ in horizontal $(z)$ direction. In comparison with two-dimensional $\mathrm{N}-V^{-}$widefield imaging, which can image micrometer-sized samples at room [29] and cryogenic temperatures [54], our approach can address even larger millimeter-sized samples by stacking many waveguides to an 2D array. Each waveguide could be accessed individually and detect local temperatures and magnetic fields. The waveguide array could be operated simultaneously, thus allowing imaging of spatially resolved magnetic field distributions and their time dynamics in extension to probing magnetism [55] or antiferromagnetic order [56] via scanning nanoprobes. By directly connecting an optical fiber bundle to the waveguide array, the device could be integrated into a photonic 
system. Moreover, integrating our device into CMOS sensors [39] could provide a perspective towards fully integrated 2D sensor arrays. Our sensing chips could take up a similar role such as CCD camera chips with applications in spatially and temporally correlated measurement in material and life sciences. Furthermore, the combination with laser-written microfluidic channels [57] could provide perspectives towards on-chip quantum sensing of liquids.

\section{METHODS}

\section{A. Waveguide fabrication}

The femtosecond laser used for waveguide writing in diamond is a $\mathrm{Yb}: \mathrm{KGW}$ fiber laser (Bluecut, Menlosystems) with 300-fs pulse duration, 515-nm wavelength (frequency doubled using an LBO crystal), focused with a 1.25 -NA oil immersion lens (RMS100×-O 100× Olympus Plan Achromat Oil Immersion Objective, $100 \times$ oil immersion, Olympus). Polished $2 \mathrm{~mm} \times 2 \mathrm{~mm} \times 0.3 \mathrm{~mm}$ synthetic single-crystal diamond samples (type II, electronic grade with nitrogen impurities $<5 \mathrm{ppb}$ ) are used. The sample is placed on a computer-controlled, threeaxis motion stage (ANT130 series, Aerotech) to translate the sample relative to the laser to form the desired photonic structures. The polarization of the incident laser is perpendicular to the scan direction.

\section{B. $\mathrm{N}-\boldsymbol{V}^{-}$-center creation}

The $\mathrm{N}-V^{-}$sensor used in this work is fabricated by ion implantation on the front facet of a commercial type IIa diamond substrate. Using a homebuilt low-energy ion implanter equipped with a Wien mass filter as well as an Einzel lens for beam focusing, nitrogen ions of energy 5 $\mathrm{keV}$ are implanted at a dose of $5 \times 10^{11}\left({ }^{15} \mathrm{~N}^{+} / \mathrm{cm}^{2}\right)$ and a beam diameter of approximately $100 \mu \mathrm{m}$. Subsequently, the substrate is annealed in ultra-high vacuum (UHV) for $3 \mathrm{~h}$ at $1000^{\circ} \mathrm{C}$ to form $\mathrm{N}-V^{-}$centers. The ramp-up time is $7\left({ }^{\circ} \mathrm{C} / \mathrm{min}\right)$ with an intermediate soak for $1 \mathrm{~h}$ at $500^{\circ} \mathrm{C}$. Between all processing steps, the substrate is boiled several hours in a 1:1:1 mixture of sulphuric, perchloric and nitric acid to remove any organic or graphitic residues from the surfaces of the diamond. Details on the annealing and ion implantation setups for sensor fabrication can be found in [58].

\section{Confocal setup}

The sample is mounted in a liquid helium, continuous flow cryostat enabling operation temperatures down to $5 \mathrm{~K}$. The temperature of the sample could further be varied and controlled with a heater which is in thermal contact with the device. The waveguides could be accessed via a high-NA (0.9) objective from one side of the diamond slab containing the $\mathrm{N}-V^{-}$centers and from the backside with a low-NA ( 0.25$)$ objective to collect the transmission through the waveguide. The objectives are mounted on movable stages for optimization of the incoupling and outcoupling. Thereby, we combined a standard confocal setup in front with additional waveguide transmission detection at the back of the sample. The characterization of the emitters could therefore be done in standard confocal spectroscopy. For measuring waveguide losses in our setup, we coupled light through the waveguides with a second low-NA objective and detect light transmitted through the waveguide at the back. The $\mathrm{N}-V^{-}$-center ensemble could also be excited directly with a high-NA objective while simultaneously reading out through the waveguide with low-NA objective. For CW ODMR measurements we spanned a wire with $20 \mu \mathrm{m}$ diameter close to the $\mathrm{N}-V^{-}$ ensemble to deliver the MW signal, which are generated by a vector signal generator (Rohde \& Schwarz SMIQ 03). Typical CW ODMR scans in our setting took $30 \mathrm{~min}$ to ensure good signal to noise. A single measurement consisted of many repeated quick scans (around $7 \mathrm{~s}$ ) over a chosen frequency range. We then averaged over the ensemble of fast scans to obtain a result for the $30 \mathrm{~min} \mathrm{CW}$ ODMR scan.

\section{ACKNOWLEDGMENTS}

The authors would like to thank Felix Breuning for experimental support in the beginning of the project. IFNCNR is grateful for technical assistance from Erasmus students Marie Dartiguelongue and Héloïse Raugel. IFNCNR and UUlm are grateful for support from the H2020 Marie Curie ITN project LasIonDef (GA n. 956387). A.K. acknowledges support of the European Regional Development Fund (EFRE) program Baden-Württemberg. M.K.K. and A.K. acknowledge support of IQst. M.H. acknowledges support from the Studienstiftung des deutschen Volkes. V.B. is thankful for financial support from the ERC project PAIDEIA GA No. 816313. A. G. is thankful for support from the Lombardy Region Project sPATIALS3, cofunded by POR FESR 2014-2020 Call HUB Ricerca e Innovazione and the H2020 Marie Curie ITN project PHOTOTRAIN (Grant No. 722591).

M.H., M.K.K., V.B., S.M.E., and A.K. conceived the project. M.H. and M.K.K. performed and analyzed all PL and ODMR measurements. V.B., J.P.H., R.Y., A.N.G., R.R., and S.M.E. produced the laser-written WG in diamond, which were implanted and annealed by J.L. and F.J. to create $\mathrm{N}-V^{-}$centers. All authors discussed the results. M.H., M.K.K., and A.K. wrote the manuscript, which was discussed and edited by all authors.

[1] M. W. Doherty, V. V. Struzhkin, D. A. Simpson, L. P. McGuinness, Y. Meng, A. Stacey, T. J. Karle, R. J. Hemley, N. B. Manson, L. C. L. Hollenberg, and S. Prawer, Electronic Properties and Metrology Applications of the 
Diamond N- $V^{-}$Center under Pressure, Phys. Rev. Lett. 112, 047601 (2014).

[2] T. Plakhotnik, M. W. Doherty, J. H. Cole, R. Chapman, and N. B. Manson, All-optical thermometry and thermal properties of the optically detected spin resonances of the nvcenter in nanodiamond, Nano. Lett. 14, 4989 (2014).

[3] J. R. Maze, P. L. Stanwix, J. S. Hodges, S. Hong, J. M. Taylor, P. Cappellaro, L. Jiang, M. G. Dutt, E. Togan, and A. Zibrov et al., Nanoscale magnetic sensing with an individual electronic spin in diamond, Nature 455, 644 (2008).

[4] J. Taylor, P. Cappellaro, L. Childress, L. Jiang, D. Budker, P. Hemmer, A. Yacoby, R. Walsworth, and M. Lukin, High-sensitivity diamond magnetometer with nanoscale resolution, Nat. Phys. 4, 810 (2008).

[5] G. Balasubramanian, I. Chan, R. Kolesov, M. Al-Hmoud, J. Tisler, C. Shin, C. Kim, A. Wojcik, P. R. Hemmer, and A. Krueger et al., Nanoscale imaging magnetometry with diamond spins under ambient conditions, Nature 455, 648 (2008).

[6] G. Kucsko, P. C. Maurer, N. Y. Yao, M. Kubo, H. J. Noh, P. K. Lo, H. Park, and M. D. Lukin, Nanometre-scale thermometry in a living cell, Nature 500, 54 (2013).

[7] S. Kolkowitz, A. Safira, A. A. High, R. C. Devlin, S. Choi, Q. P. Unterreithmeier, D. Patterson, A. S. Zibrov, V. E. Manucharyan, H. Park, and M. D. Lukin, Probing johnson noise and ballistic transport in normal metals with a single-spin qubit, Science 347, 1129 (2015).

[8] I. Lovchinsky, J. D. Sanchez-Yamagishi, E. K. Urbach, S. Choi, S. Fang, T. I. Andersen, K. Watanabe, T. Taniguchi, A. Bylinskii, E. Kaxiras, P. Kim, H. Park, and M. D. Lukin, Magnetic resonance spectroscopy of an atomically thin material using a single-spin qubit, Science 355, 503 (2017).

[9] C. Degen, Scanning magnetic field microscope with a diamond single-spin sensor, Appl. Phys. Lett. 92, 243111 (2008).

[10] P. Maletinsky, S. Hong, M. S. Grinolds, B. Hausmann, M. D. Lukin, R. L. Walsworth, M. Loncar, and A. Yacoby, A robust scanning diamond sensor for nanoscale imaging with single nitrogen-vacancy centres, Nat. Nanotechnol. 7, 320 (2012).

[11] M. Atatüre, D. Englund, N. Vamivakas, S.-Y. Lee, and J. Wrachtrup, Material platforms for spin-based photonic quantum technologies, Nat. Rev. Mater. 3, 38 (2018).

[12] D. D. Awschalom, R. Hanson, J. Wrachtrup, and B. B. Zhou, Quantum technologies with optically interfaced solid-state spins, Nat. Photonics 12, 516 (2018).

[13] Y. Ruan, D. A. Simpson, J. Jeske, H. EbendorffHeidepriem, D. W. Lau, H. Ji, B. C. Johnson, T. Ohshima, S. Afshar, and L. Hollenberg et al., Magnetically sensitive nanodiamond-doped tellurite glass fibers, Sci. Rep. 8, 1 (2018).

[14] D. Bai, M. H. Huynh, D. A. Simpson, P. Reineck, S. A. Vahid, A. D. Greentree, S. Foster, H. EbendorffHeidepriem, and B. C. Gibson, Fluorescent diamond microparticle doped glass fiber for magnetic field sensing, APL Mater. 8, 081102 (2020).

[15] L. Hanlon, V. Gautam, J. D. A. Wood, P. Reddy, M. S. J. Barson, M. Niihori, A. R. J. Silalahi, B. Corry, J. Wrachtrup, M. J. Sellars, V. R. Daria, P. Maletinsky, G. J.
Stuart, and M. W. Doherty, Diamond nanopillar arrays for quantum microscopy of neuronal signals, Neurophotonics 7, 035002 (2020).

[16] T. M. Babinec, B. J. M. Hausmann, M. Khan, Y. Zhang, J. R. Maze, P. R. Hemmer, and M. Loncar, A diamond nanowire single-photon source, Nat. Nanotechnol. 5, 195 (2010).

[17] D. Englund, B. Shields, K. Rivoire, F. Hatami, J. Vuckovic, H. Park, and M. D. Lukin, Deterministic coupling of a single nitrogen vacancy center to a photonic crystal cavity, Nano Lett. 10, 3922 (2010).

[18] K. G. Fehler, A. P. Ovvyan, N. Gruhler, W. H. P. Pernice, and A. Kubanek, Efficient coupling of an ensemble of nitrogen vacancy center to the mode of a high-Q, $\mathrm{Si}_{3} \mathrm{~N}_{4}$ photonic crystal cavity, ACS Nano 13, 6891 (2019).

[19] J. Olthaus, P. P. J. Schrinner, D. E. Reiter, and C. Schuck, Optimal photonic crystal cavities for coupling nanoemitters to photonic integrated circuits, Adv. Quantum Technol. 3, 1900084 (2020).

[20] B. Sotillo, V. Bharadwaj, J. P. Hadden, M. Sakakura, A. Chiappini, T. T. Fernandez, S. Longhi, O. Jedrkiewicz, Y. Shimotsuma, L. Criante, R. Osellame, G. Galzerano, M. Ferrari, K. Miura, R. Ramponi, P. E. Barclay, and S. M. Eaton, Diamond photonics platform enabled by femtosecond laser writing, Sci. Rep. 6, 35566 (2016).

[21] S. M. Eaton, J. P. Hadden, V. Bharadwaj, J. Forneris, F. Picollo, F. Bosia, B. Sotillo, A. N. Giakoumaki, O. Jedrkiewicz, A. Chiappini, M. Ferrari, R. Osellame, P. E. Barclay, P. Olivero, and R. Ramponi, Quantum micro-nano devices fabricated in diamond by femtosecond laser and ion irradiation, Adv. Quantum Technol. 2, 1900006 (2019).

[22] T. Schröder, M. E. Trusheim, M. Walsh, L. Li, J. Zheng, M. Schukraft, A. Sipahigil, R. E. Evans, D. D. Sukachev, and C. T. Nguyen et al., Scalable focused ion beam creation of nearly lifetime-limited single quantum emitters in diamond nanostructures, Nat. Commun. 8, 1 (2017).

[23] Y.-C. Chen, P. S. Salter, S. Knauer, L. Weng, A. C. Frangeskou, C. J. Stephen, S. N. Ishmael, P. R. Dolan, S. Johnson, B. L. Green, G. W. Morley, M. E. Newton, J. G. Rarity, M. J. Booth, and J. M. Smith, Laser writing of coherent colour centres in diamond, Nat. Photonics 11, 77 (2017).

[24] J. P. Hadden, V. Bharadwaj, B. Sotillo, S. Rampini, R. Osellame, J. D. Witmer, H. Jayakumar, T. T. Fernandez, A. Chiappini, C. Armellini, M. Ferrari, R. Ramponi, P. E. Barclay, and S. M. Eaton, Integrated waveguides and deterministically positioned nitrogen vacancy centers in diamond created by femtosecond laser writing, Optics Lett. 43, 3586 (2018).

[25] Y.-C. Chen, B. Griffiths, L. Weng, S. S. Nicley, S. N. Ishmael, Y. Lekhai, S. Johnson, C. J. Stephen, B. L. Green, G. W. Morley, M. E. Newton, M. J. Booth, P. S. Salter, and J. M. Smith, Laser writing of individual nitrogen-vacancy defects in diamond with near-unity yield, Optica 6, 662 (2019).

[26] J. L. Webb, J. D. Clement, L. Troise, S. Ahmadi, G. J. Johansen, A. Huck, and U. L. Andersen, Nanotesla sensitivity magnetic field sensing using a compact diamond nitrogen-vacancy magnetometer, Appl. Phys. Lett. 114, 231103 (2019). 
[27] N. Arunkumar, D. B. Bucher, M. J. Turner, P. TomHon, D. Glenn, S. Lehmkuhl, M. D. Lukin, H. Park, M. S. Rosen, T. Theis, and R. L. Walsworth, Micron-scale nv-nmr spectroscopy with signal amplification by reversible exchange, PRX Quantum 2, 010305 (2021).

[28] T. Van der Sar, F. Casola, R. Walsworth, and A. Yacoby, Nanometre-scale probing of spin waves using single electron spins, Nat. Commun. 6, 1 (2015).

[29] J.-P. Tetienne, N. Dontschuk, D. A. Broadway, A. Stacey, D. A. Simpson, and L. C. L. Hollenberg, Quantum imaging of current flow in graphene, Sci. Adv. 3, e1602429 (2017).

[30] B. Sotillo, A. Chiappini, V. Bharadwaj, J. P. Hadden, F. Bosia, P. Olivero, M. Ferrari, R. Ramponi, P. E. Barclay, and S. M. Eaton, Polarized micro-raman studies of femtosecond laser written stress-induced optical waveguides in diamond, Appl. Phys. Lett. 112, 031109 (2018).

[31] F. Jelezko, T. Gaebel, I. Popa, A. Gruber, and J. Wrachtrup, Observation of Coherent Oscillations in a Single Electron Spin, Phys. Rev. Lett. 92, 076401 (2004).

[32] Y. Kubo, F. R. Ong, P. Bertet, D. Vion, V. Jacques, D. Zheng, A. Dréau, J.-F. Roch, A. Auffeves, F. Jelezko, J. Wrachtrup, M. F. Barthe, P. Bergonzo, and D. Esteve, Strong Coupling of a Spin Ensemble to a Superconducting Resonator, Phys. Rev. Lett. 105, 140502 (2010).

[33] M. W. Doherty, F. Dolde, H. Fedder, F. Jelezko, J. Wrachtrup, N. B. Manson, and L. C. L. Hollenberg, Theory of the ground-state spin of the $\mathrm{N}-V^{-}$center in diamond, Phys. Rev. B 85, 205203 (2012).

[34] D. Budker and M. Romalis, Optical magnetometry, Nat. Phys. 3, 227 (2007).

[35] B. Sotillo, V. Bharadwaj, J. Hadden, S. Rampini, A. Chiappini, T. Fernandez, C. Armellini, A. Serpengüzel, M. Ferrari, P. Barclay, R. Ramponi, and S. Eaton, Visible to infrared diamond photonics enabled by focused femtosecond laser pulses, Micromachines 8, 60 (2017).

[36] Y. Matsuzaki, H. Morishita, T. Shimooka, T. Tashima, K. Kakuyanagi, K. Semba, W. Munro, H. Yamaguchi, N. Mizuochi, and S. Saito, Optically detected magnetic resonance of high-density ensemble of nv- centers in diamond, J. Phys.: Condens. Matter 28, 275302 (2016).

[37] A. Kuwahata, T. Kitaizumi, K. Saichi, T. Sato, R. Igarashi, T. Ohshima, Y. Masuyama, T. Iwasaki, M. Hatano, and F. Jelezko et al., Magnetometer with nitrogen-vacancy center in a bulk diamond for detecting magnetic nanoparticles in biomedical applications, Sci. Rep. 10, 1 (2020).

[38] L. Rondin, J.-P. Tetienne, T. Hingant, J.-F. Roch, P. Maletinsky, and V. Jacques, Magnetometry with nitrogenvacancy defects in diamond, Rep. Progress Phys. 77, 056503 (2014).

[39] D. Kim, M. I. Ibrahim, C. Foy, M. E. Trusheim, R. Han, and D. R. Englund, A cmos-integrated quantum sensor based on nitrogen-vacancy centres, Nat. Electron. 2, 284 (2019).

[40] E. V. Levine, M. J. Turner, P. Kehayias, C. A. Hart, N. Langellier, R. Trubko, D. R. Glenn, R. R. Fu, and R. L. Walsworth, Principles and techniques of the quantum diamond microscope, Nanophotonics 8, 1945 (2019).

[41] See Supplemental Material at http://link.aps.org/supple mental/10.1103/PhysRevApplied.15.054059 for details on the measurement.
[42] V. M. Acosta, E. Bauch, M. P. Ledbetter, A. Waxman, L.-S. Bouchard, and D. Budker, Temperature Dependence of the Nitrogen-Vacancy Magnetic Resonance in Diamond, Phys. Rev. Lett. 104, 070801 (2010).

[43] M. W. Doherty, V. M. Acosta, A. Jarmola, M. S. J. Barson, N. B. Manson, D. Budker, and L. C. L. Hollenberg, Temperature shifts of the resonances of the $\mathrm{nv}^{-}$center in diamond, Phys. Rev. B 90, 041201(R) (2014).

[44] T. Wolf, P. Neumann, K. Nakamura, H. Sumiya, T. Ohshima, J. Isoya, and J. Wrachtrup, Subpicotesla Diamond Magnetometry, Phys. Rev. X 5, 041001 (2015).

[45] P. Balasubramanian, C. Osterkamp, Y. Chen, X. Chen, T. Teraji, E. Wu, B. Naydenov, and F. Jelezko, Dc magnetometry with engineered nitrogen-vacancy spin ensembles in diamond, Nano Lett. 19, 6681 (2019).

[46] I. Fescenko, A. Jarmola, I. Savukov, P. Kehayias, J. Smits, J. Damron, N. Ristoff, N. Mosavian, and V. M. Acosta, Diamond magnetometer enhanced by ferrite flux concentrators, Phys. Rev. Res. 2, 023394 (2020).

[47] D. R. Glenn, D. B. Bucher, J. Lee, M. D. Lukin, H. Park, and R. L. Walsworth, High-resolution magnetic resonance spectroscopy using a solid-state spin sensor, Nature $\mathbf{5 5 5}$, 351 (2018).

[48] K. Mizuno, M. Nakajima, H. Ishiwata, Y. Masuyama, T. Iwasaki, and M. Hatano, Wide-field diamond magnetometry with millihertz frequency resolution and nanotesla sensitivity, AIP Adv. 8, 125316 (2018).

[49] F. M. Stürner, A. Brenneis, T. Buck, J. Kassel, R. Rölver, T. Fuchs, A. Savitsky, D. Suter, J. Grimmel, S. Hengesbach, M. Förtsch, K. Nakamura, H. Sumiya, S. Onoda, J. Isoya, and F. Jelezko, Integrated and portable magnetometer based on nitrogen-vacancy ensembles in diamond, Adv. Quantum Technol. 4, 2000111 (2021).

[50] D. J. McCloskey, N. Dontschuk, D. A. Broadway, A. Nadarajah, A. Stacey, J.-P. Tetienne, L. C. L. Hollenberg, S. Prawer, and D. A. Simpson, Enhanced widefield quantum sensing with nitrogen-vacancy ensembles using diamond nanopillar arrays, ACS Appl. Mater. Interfaces 12, 13421 (2020).

[51] T. Kononenko, V. Konov, S. Pimenov, N. Rossukanyi, A. Rukovishnikov, and V. Romano, Three-dimensional laser writing in diamond bulk, Diamond Relat. Mater. 20, 264 (2011).

[52] A. Courvoisier, M. J. Booth, and P. S. Salter, Inscription of 3D waveguides in diamond using an ultrafast laser, Appl. Phys. Lett. 109, 031109 (2016).

[53] J. Gong, K. Ansari, and J. A. van Kan, Design and fabrication of three-dimensional single-mode waveguide arrays in bulk diamond through direct focused proton beam implantation, Opt. Mater. 99, 109527 (2020).

[54] D. A. Broadway, S. C. Scholten, C. Tan, N. Dontschuk, S. E. Lillie, B. C. Johnson, G. Zheng, Z. Wang, A. R. Oganov, S. Tian, C. Li, H. Lei, L. Wang, L. C. L. Hollenberg, and J.-P. Tetienne, Imaging domain reversal in an ultrathin van der waals ferromagnet, Adv. Mater. 32, 2003314 (2020).

[55] L. Thiel, Z. Wang, M. Tschudin, D. Rohner, I. GutiérrezLezama, N. Ubrig, M. Gibertini, E. Giannini, A. Morpurgo, and P. Maletinsky, Probing magnetism in $2 \mathrm{~d}$ materials at the nanoscale with single spin microscopy, Science 364, 973 (2019). 
[56] I. Gross, W. Akhtar, V. Garcia, L. J. Martínez, S. Chouaieb, K. Garcia, C. Carrétéro, A. Barthélémy, P. Appel, P. Maletinsky, J.-V. Kim, J. Y. Chauleau, N. Jaouen, M. Viret, M. Bibes, A. Fusil, and V. Jacques, Real-space imaging of non-collinear antiferromagnetic order with a single-spin magnetometer, Nature 549, 252 (2017).

[57] V. Bharadwaj, O. Jedrkiewicz, J. P. Hadden, B. Sotillo, M. R. Vázquez, P. Dentella, T. T. Fernandez, A. Chiappini, A.
N. Giakoumaki, T. Le Phu, M. Bollani, M. Ferrari, R. Ramponi, P. E. Barclay, and S. M. Eaton, Femtosecond laser written photonic and microfluidic circuits in diamond, J. Phys.: Photonics 1, 022001 (2019).

[58] J. Lang, S. Häußler, J. Fuhrmann, R. Waltrich, S. Laddha, J. Scharpf, A. Kubanek, B. Naydenov, and F. Jelezko, Long optical coherence times of shallow-implanted, negatively charged silicon vacancy centers in diamond, Appl. Phys. Lett. 116, 064001 (2020). 